\title{
Taxa de prenhez de vacas Nelore lactantes tratadas com progesterona associada à remoção temporária de bezerros ou aplicação de gonadotrofina coriônica eqüina
}

\author{
Ronaldo Luiz Ereno ${ }^{1}$, Thales Ricardo Rigo Barreiros ${ }^{2}$, Marcelo Marcondes Seneda ${ }^{3}$, Pietro \\ Sampaio Baruselli4, Marcelo Figueira Pegorer ${ }^{1}$, Ciro Moraes Barros ${ }^{1}$
}

\footnotetext{
${ }^{1}$ Departamento de Farmacologia, Instituto de Biociências - UNESP, Botucatu - SP.

2 Departamento de Reprodução Animal, Faculdades Integradas de Campo Mourão - PR.

${ }^{3}$ Departamento de Clínicas Veterinárias - Centro de Ciências Agrárias UEL, Londrina - PR.

${ }^{4}$ Departamento de Reprodução Animal, FMVZ-USP, São Paulo.
}

RESUMO - Avaliaram-se os efeitos da remoção temporária de bezerros (RTB) ou da aplicação de gonadotrofina coriônica eqüina (eCG) na taxa de prenhez (TP) de vacas Nelore lactantes tratadas com um dispositivo intravaginal liberador de progesterona (DILP). No experimento 1, 83 vacas Nelore e 102 mestiças (Nelore $\times$ Red Angus) foram distribuídas em três grupos. Em dia aleatório do ciclo estral (D0), os animais do grupo 1 foram tratados com benzoato de estradiol (BE; $2,5 \mathrm{mg}$, IM, Estrogin ${ }^{\circledR}$ ) e um DILP (1 g de progesterona, DIB $\left.{ }^{\circledR}\right)$, removido no D9, quando também se administrou d-cloprostenol $\left(150 \mu \mathrm{g}, \mathrm{IM}\right.$, Prolise $\left.{ }^{\circledR}\right)$ e aproximadamente 12 horas após identificação do estro realizou-se a inseminação artificial. No grupo 2 (IATF), o tratamento foi semelhante ao aplicado no grupo 1, porém, administrou-se uma segunda dose de benzoato de estradiol (1 mg) no D10 e 30-36 horas mais tarde realizou-se a inseminação artificial em tempo fixo (IATF). No grupo 3 (IATF/RTB), os bezerros foram removidos a partir do D9 até a inseminação artificial com tempo fixo (IATF). As taxas de prenhez para as vacas Nelore e mestiças foram, respectivamente, de 7,69 e 41\% (Controle); 23,52 e 59,57\% (IATF); 69,44 e 55,81\% (IATF/RTB). No experimento 2, 255 vacas Nelore lactantes foram distribuídas em três grupos. Os animais do grupo 4 (IATF) foram tratados com benzoato de estradiol (2 mg) e um DILP (1,9 g de progesterona, CIDR-B $\left.{ }^{\circledR}\right)$ no D0 e no D8 sofreram remoção do DILP no D8 e administração de $25 \mathrm{mg}$ de dinoprost (IM, Lutalyse $\left.{ }^{\circledR}\right)$. No D9, foi aplicado benzoato de estradiol $(1 \mathrm{mg})$ realizando-se a IATF 30-36 horas mais tarde. No grupo 5 (IATF/RTB), os bezerros foram removidos no D9 até a IATF. O grupo 6 (eCG) foi semelhante ao IATF, exceto pela aplicação de eCG no D9 (400 UI, IM, Novormon ${ }^{\circledR}$ ). As taxas de prenhez foram de 50,57 (IATF), 53,57 (IATF/RTB) e 54,76\% (eCG). A associação de RTB ao tratamento hormonal com DILP aumenta as taxas de prenhez, enquanto a adição de eCG ao tratamento não melhora as taxa de prenhez de vacas Nelore lactantes ciclando e em boa condição corporal.

Palavras-chave: eCG, inseminação artificial, bovinos, progesterona, remoção de bezerros

\section{Pregnancy rates in milking Nelore cows submitted to progesterone treatment associated to temporary calf removal or eCG administration}

\footnotetext{
ABSTRACT - This study aimed to evaluate the effects of temporary calf removal (TCR) or eCG administration on pregnancy rates of milking Nelore cows treated with a intravaginal progesterone releasing device (IPRD). In the first experiment 83 Nelore and 102 Nelore vs. Red Angus crossbred cows were randomly allocated to 3 groups. Animals in Group $1(\mathrm{CON})$ received estradiol benzoate $\left(\mathrm{EB} ; 2.5 \mathrm{mg}\right.$, i.m., Estrogin $\left.{ }^{\circledR}\right)$ and an IPRD (1g progesterone, DIB $\left.{ }^{\circledR}\right)$ in a random stage of the estrous cycle (D0). At D9 the IPRD was removed and the animals were treated with d-cloprostenol $\left(150 \mu \mathrm{g}\right.$, i.m., Prolise $\left.{ }^{\mathbb{R}}\right)$ The animals were artificially inseminated (AI) $12 \mathrm{~h}$ after estrus detection. Group 2 (FTAI) was similar to CON, except that another dose of EB $(1 \mathrm{mg})$ was administered at D10, and 30-36 hours later artificial insemination (FTAI) was performed at a fixed time. In Group 3 (FTAI/TCR) the calves were removed from D9 to the FTAI. Pregnancy rates for Nelore and crossbred cows were, respectively, 7.69 and $41 \%(\mathrm{CON}) ; 23.52$ and 59.57 (FTAI); 69.44 and 55.81 (FTAI/TCR). In the second experiment, 255 lactating Nelore cows were allocated to 3 groups. At D0, animals in Group 4 (FTAI) received EB (2 mg) and one IPRD ( $1.9 \mathrm{~g}$ progesterone, CIDR- $\left.{ }^{\circledR}\right)$, which was removed at D8 when the cows received $25 \mathrm{mg}$ of dinoprost (i.m. Lutalyse $\left.^{\circledR}\right)$. At D9 EB $(1 \mathrm{mg})$ was administered and 30-36 hours later all animas were FTAI. In Group 5 (FTAI/TCR) the calves were removed from D9 to FTAI. The Group 6 (eCG) was similar to IATF except for the administration of eCC in D9 (400 IU, i.m., Novormon ${ }^{\circledR}$ ). Pregnancy rates were: $50.57 \%$ (FTAI), $53.57 \%$ (FTAI/TCR) and $54.76 \%$ (eCG). These results indicated that TCR associated with IPRD increase pregnancy rates, whereas the addition of eCG to IPRD do not improve pregnancy rates in Nelore cows which are cycling and in lactation with good body condition.
}

Key Words: artificial insemination, bovine, calf removal, eCG, progesterone 


\section{Introdução}

A pecuária de corte nacional ainda apresenta baixos índices produtivos por continuar sendo conduzida como uma atividade extrativista ou que demanda baixo uso de insumos e biotecnologias. No atual cenário mundial, essa característica da bovinocultura brasileira precisa ser modificada para que a atividade se torne rentável e competitiva (Pires et al., 2004).

No Brasil, a tendência de valorização das áreas agricultáveis exercerá pressão sobre as áreas de pastagem. Estima-se que nos próximos dez anos cerca de 24 milhões de hectares de pastagens serão substituídos por lavouras de grãos. Entretanto, a previsão de crescimento do rebanho nacional nos próximos dez anos é de 170 milhões de cabeças em 2003 para 181,8 milhões de cabeças em 2012 (Anualpec, 2003). Neste caso, a pecuária de corte nacional terá que se apoiar não apenas na capacidade de suporte das pastagens como também no aumento da eficiência reprodutiva. As principais alternativas para se aumentar o desempenho reprodutivo de vacas de corte seriam elevar o número de vacas com estro no início da estação de monta e aumentar a taxa de concepção no primeiro serviço (Wiltbank, 1970).

Considerando os índices zootécnicos da pecuária nacional, constata-se que o Brasil ainda está longe de alcançar as metas citadas, pois seu rebanho de corte apresenta intervalo de partos médio de 21 meses (Zimmer \& Euclides Filho, 1997). O intervalo de partos depende da duração do anestro pós-parto e da condição intimamente relacionada ao estímulo da mamada (quantidade e freqüência), segundo Bellows \& Short (1994), a qual impede a retomada do padrão de secreção pulsátil de LH, capaz de dar suporte ao desenvolvimento final e à maturação do folículo pré-ovulatório (Willians \& Griffith, 1995). A ausência da pulsatilidade de LH é provocada pela retroalimentação negativa dos estrógenos ovarianos na secreção pulsátil de GnRH pelo hipotálamo (Walters et al., 1982; Wiltbank et al., 2002).

A ausência da pulsatilidade de LH no início da parição (primeiros 30 dias) é atribuída à depleção nas concentrações dessa gonadotrofina na hipófise anterior (Nett et al., 1987). Após o restabelecimento desses estoques, o desmame completo ou o temporário (48 ou 96 horas) aumentam a freqüência dos pulsos de LH (Dunlap et al., 1981, Shively \& Williams, 1989), seguido pelo aumento de receptores foliculares para LH e FSH (Walters et al., 1982) e, posteriormente, pela ovulação induzida pelo pico de LH (Zollers et al., 1991).

Além da restrição da mamada, a utilização de gonadotrofina coriônica eqüina $(\mathrm{eCG})$ tem se mostrado benéfica no desenvolvimento folicular de vacas em anestro pós-parto por se ligar aos receptores de FSH e LH (Kastelic et al., 1999; Yavas \& Walton, 2000a).

Em razão da facilidade de adaptação ao clima tropical (adaptabilidade e rusticidade), da boa resistência aos endo e ectoparasitas e da estrutura física que lhe proporciona facilidade de locomoção, além de excelente habilidade materna (facilidade de parto e proteção da cria), os zebuínos (Bos taurus indicus) predominam em países do hemisfério sul (Santiago, 1985; Santos, 1995; Bó et al., 2003). Entre as raças de corte, a Nelore possui o maior contigente numérico (90 milhões de cabeças do total de 170 milhões) do rebanho nacional, sendo considerada o alicerce da cadeia produtiva pecuária de corte brasileira (ACNB, 2006). De acordo com a Associação Brasileira dos Criadores de Zebu (ABCZ, 2003), apenas 4,6\% dos rebanhos de corte e de leite brasileiros são inseminados artificialmente (IA). Apesar de esta biotecnologia ser fundamental para o melhoramento genético do rebanho bovino, a identificação do estro continua a ser um fator limitante para sua ampla utilização (Macmillan, 1992).

Em fêmeas Nelore, o anestro pós-parto pode ser mais acentuado pela maior freqüência do ato da mamada de seus bezerros, em média oito vezes ao dia (Cubas et al., 1985). Além disso, a curta duração do estro (aproximadamente 11 horas), associada à alta incidência de estros noturnos (30-50\%), dificulta a identificação do estro e prejudica a implantação de programas convencionais de inseminação artificial (Barros et al., 1998; Barros \& Fernandes, 1998). Uma das formas de contornar esse problema é desenvolver protocolos de sincronização da ovulação que permitam realizar a inseminação artificial em tempo fixo (IATF), sem necessidade de observação de cio, durante o período de tratamento.

Entre os protocolos para sincronização da ovulação e IATF, os mais utilizados, principalmente para animais em anestro pós-parto e com boa condição corporal, são aqueles que associam uma fonte de progesterona/progestágeno à aplicação de estrógenos e/ou outros hormônios (Bó et al., 2003; Baruselli et al., 2004).

Neste trabalho, objetivou-se verificar se a remoção temporária de bezerros (RTB) ou a utilização de eCG melhoram a taxa de prenhez de vacas Nelore lactantes tratadas com dispositivo intravaginal liberador de progesterona e inseminadas em tempo fixo.

\section{Material e Métodos}

Foram utilizadas vacas da raça Nelore e mestiças (Nelore $\times$ Red Angus) com 60 a 90 dias pós-parto e escore de 
condição corporal de 2,5 a 3,5 (1 = muito magra a $5=$ muito gorda), segundo Lowman et al. (1976). Os animais foram manejados extensivamente em pastagens de Brachiaria brizantha e receberam suplementação mineral ad libitum.

Os experimentos foram realizados no período de janeiro a março, em propriedades particulares, nos municípios de Pratânia-SP (Fazenda Palmeira da Serra, experimento 1) e de Santa Rita do Pardo-MS (Fazenda Campanário, experimento 2).

O diagnóstico de gestação foi realizado por meio de ultra-sonografia (Aloka SSD 500, $5 \mathrm{mHZ}$ ) 40 a 50 dias após a inseminação artificial para determinação da taxa de prenhez (número de fêmeas prenhes em relação ao número de fêmeas tratadas).

As inseminações realizadas no experimento 1 foram feitas por apenas um inseminador utilizando-se sêmen de três touros da raça Nelore na inseminação artificial, distribuído igualmente entre os grupos tratados.

No experimento 2, utilizou-se sêmen de 2 a 3 touros por réplica do experimento, avaliados previamente pelo teste de termorresistência rápida, descrito no Manual para exame Andrológico e avaliação de sêmen animal (CBRA, 1998). O sêmen era proveniente de diversas centrais nacionais e de uma mesma partida de congelamento para cada touro utilizado. A distribuição dos touros por bloco foi feita de forma equiparada para cada grupo e os dois inseminadores permaneceram em esquema de revezamento a cada 40 vacas. Todo sêmen estava acondicionado em palhetas e armazenado em botijões de nitrogênio líquido. O sêmen foi descongelado com a utilização de um aparelho banho-maria com temperatura de $37^{\circ} \mathrm{C}$ por aproximadamente 30 segundos. Foi utilizada a técnica de inseminação artificial profunda descrita por Mies Filho (1977).

No experimento 1, 230 vacas Nelore (variedade pele rosa) e mestiças (Nelore $\times$ Red Angus) com 60 a 90 dias pósparto, condição corporal e período pós-parto semelhantes foram submetidas a exame ultra-sonográfico transretal. Foram selecionados apenas os animais com corpo lúteo (CL) e/ou presença de folículos em pelo menos um dos ovários; vacas com ovários lisos e pequenos não foram incluídas no experimento.

As vacas selecionadas (185 vacas, 102 mestiças e 83 Nelore) foram distribuídas ao acaso em três grupos experimentais: controle (25 vacas, 13 Nelore e 12 mestiças), IATF (81 vacas; 34 Nelore e 47 mestiças) e IATF-RTB (remoção temporária de bezerros; 79 vacas, 36 Nelore e 43 mestiças).

Em dia aleatório do ciclo estral (D0), os animais receberam um dispositivo intravaginal contendo progesterona
( $1 \mathrm{~g}$ de progesterona, DIB $^{\circledR}$ ) e uma injeção de benzoato de estradiol (BE, 2,5 mg, Estrogin ${ }^{\circledR}$, via IM, D0). Nove dias depois, o dispositivo intravaginal foi retirado e os animais foram tratados com $\mathrm{PGF}_{2} \alpha$ (d-cloprostenol, $150 \mu \mathrm{g}$, Prolise ${ }^{\circledR}$, via IM). Após a administração, os animais do grupo controle foram monitorados durante cinco dias consecutivos, duas vezes ao dia, para identificação do estro na presença de rufiãs (fêmeas androgenizadas com Decadurabolin ${ }^{\circledR}$, providas de buçal marcador, na proporção de uma rufiã para sete vacas, grupo controle), e inseminados 12 horas após identificação do estro.

Grupo controle (Control group)

$\mathrm{D} 0$

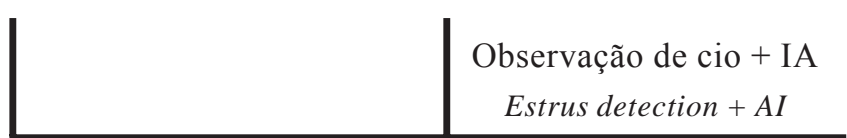

$\downarrow$ DIB

$\downarrow$ DIB

$\mathrm{BE}(2,5 \mathrm{mg}) \quad$ PGF2 $\alpha$

Nas vacas do grupo IATF, o tratamento foi similar ao do grupo controle, porém, 24 horas após a retirada do dispositivo intravaginal e aplicação de PGF2 $\alpha$, todos os animais receberam uma injeção de BE (1,0 mg, via IM, D10) e 36 horas mais tarde foram inseminados sem observação de cio(IATF).

Grupo (Group) IATF

\begin{tabular}{|lccr} 
D0 & D9 & D10 & D11 \\
& & $24 \mathrm{~h}$ & $30-36 \mathrm{~h}$ \\
$\downarrow$ DIB & $\downarrow$ DIB & BE & IATF \\
BE $(2,5 \mathrm{mg})$ & PGF2 $\alpha$ & $(1 \mathrm{mg})$ &
\end{tabular}

Nas vacas do grupo IATF/RTB, o tratamento foi similar ao do grupo IATF, exceto pela remoção temporária dos bezerros no D9 (54 h) até o dia da IATF (D11).

\section{Grupo (Group) IATF/RTB}

RTB - $54 \mathrm{~h}$

\begin{tabular}{|cccr} 
DO & D9 & D10 & D11 \\
& & $24 \mathrm{~h}$ & $30-36 \mathrm{~h}$ \\
\hline $\mathrm{DIB}$ & $\begin{array}{l} \\
\mathrm{BE}(2,5 \mathrm{mg})\end{array}$ & $\mathrm{BE}$ & $\mathrm{IATF}$ \\
& $\mathrm{PGF} 2 \alpha$ & $(1 \mathrm{mg})$ & $\begin{array}{r}\text { Retorno } \\
\text { bezerros }\end{array}$ \\
& & & (Calf return)
\end{tabular}


No experimento 2, foram utilizadas 255 vacas lactantes e pluríparas da raça Nelore, com 41 a 65 dias pós-parto. Os animais foram submetidos a exame por palpação retal para seleção daqueles com corpo lúteo (CL) e/ou flutuação (presença de folículos) em pelo menos um dos ovários. As vacas com ovários lisos e pequenos foram retiradas do experimento. O experimento foi conduzido em duas réplicas, de acordo com as datas de parição dos animais; a primeira foi realizada em 06/01/2003 e a segunda, em 01/02/2003. Como as datas não influenciaram os resultados, os dados das duas réplicas foram agrupados.

Os animais foram distribuídos em três grupos experimentais, grupo IATF - controle (97 vacas), grupo IATF/ RTB (84 vacas) e grupo IATF/eCG (84 vacas), de forma equiparada em relação à condição corporal e ao período pós-parto, compondo grupos com número aproximadamente igual de animais.

Em dia aleatório do ciclo estral, os animais receberam um dispositivo intravaginal contendo progesterona $(1,9 \mathrm{~g}$ de progesterona, CIDR $^{\circledR}$ ) e uma injeção de benzoato de estradiol (benzoato de estradiol, $2 \mathrm{mg}$, Estrogin ${ }^{\circledR}$, via IM, D0). Oito dias depois, o dispositivo intravaginal foi retirado e os animais receberam uma dose de $\mathrm{PGF}_{2} \alpha$ (dinaprost trometamina, $25 \mathrm{mg}$, Lutalyse ${ }^{\circledR}$, via IM). Vinte e quatro horas depois, uma injeção de benzoato de estradiol (1,0 mg, via IM, D9) foi aplicada e 30 a 36 horas mais tarde todos os animais foram inseminados sem observação de estro (IATF).

Nas vacas do grupo IATF/RTB, o tratamento foi similar ao do grupo IATF-controle, exceto pela remoção temporária dos bezerros no dia 8 ( 54 horas) até o dia da IATF (dia 10).

Nas vacas tratadas com gonadotrofina coriônica eqüina (Grupo IATF/eCG), o tratamento foi similar ao do grupo controle, porém, no momento da retirada do dispositivo intravaginal e aplicação de PGF2 $\alpha$, todos os animais receberam uma injeção de 400 UI de eCG (Novormon ${ }^{\circledR}$, via IM, D8) e foram inseminados 30 a 36 horas mais tarde, sem observação do estro (IATF).

Os resultados obtidos no experimento 1 foram analisados por meio de regressão logística utilizando-se o PROC GENMOD do programa SAS (SAS, 1999) e considerando no modelo os efeitos dos touros, da raça das vacas e do escore de condição corporal. As comparações dos resultados foram feitas por meio de contraste ortogonais entre os grupos de cada raça. No experimento 2 as taxas de prenhez foram comparadas utilizando-se o teste qui-quadrado corrigido de Yates $\left(\chi^{2}\right)$, a 5\% de significância.

\section{Resultados e Discussão}

Como houve influência da interação raça $\times$ grupos de tratamentos $(\mathrm{P}=0,002)$ sobre a manifestação de estro e a taxa de prenhez e, entre as vacas Nelore (Tabela 1), as taxas de prenhez foram menores nos tratamentos controle $(7,69 \%)$ e IATF $(23,52 \%)$ que no tratamento IATF-RTB $(69,44 \%)$, os grupos foram comparados dentro de cada raça. Quando o desempenho da mesma raça foi comparado entre os três tratamentos hormonais, a raça Nelore diferiu estatisticamente entre os grupos controle e IATF $(\mathrm{P}=0,004)$ e entre os grupos IATF e IATF-RTB $(\mathrm{P}=0,001)$, o que não ocorreu com as vacas mestiças $(\mathrm{P}=0,34)$.

As vacas mestiças do grupo controle $(\mathrm{P}=0,079)$ e IATF $(\mathrm{P}=0,001)$ responderam melhor aos tratamentos em relação às Nelore. Porém, as vacas Nelore responderam melhor ao tratamento IATF/RTB que as mestiças $(\mathrm{P}=0,019$, Tabela 1$)$. Não houve interferência de touros, inseminador e escore corporal nos resultados.

No experimento 2 não houve influência da condição corporal ou do intervalo pós-parto (41 a 65 dias) nas taxas de prenhez dos três grupos experimentais (Tabela 2 ).

Ressalta-se que, nos experimentos 1 e 2, não houve rejeição de bezerros após o desmame temporário (Grupo IATF/RTB).

Comparou-se a eficiência de tratamentos hormonais (dispositivos intravaginais liberadores de progesterona e eCG) que potencialmente induzem o ciclo estral de vacas em anestro pós-parto associados à remoção temporária de bezerro, que também facilita o retorno à ciclicidade de vacas em anestro pós-parto. À exceção das vacas Nelore do experimento 1 , tanto a remoção do bezerro quanto o uso de eCG não melhoraram as taxas de prenhez de vacas Nelore lactantes tratadas com dispositivo intravaginal liberador de progesterona.

As concentrações de progesterona liberadas por dispositivos intravaginais mantiveram-se abaixo das encontradas na fase luteal, o que permite aumento na pulsatilidade de LH, ocasionando maior crescimento do folículo dominante e, conseqüentemente, maior produção de estradiol, o que resulta na ovulação do folículo em 75 a $100 \%$ das vacas após a retirada do dispositivo (Savio et al., 1993; Yavas \& Walton, 2000a). Outro efeito benéfico da progesterona exógena na vaca em anestro pós-parto é o de prolongar a vida do corpo lúteo formado a partir do folículo selecionado neste período, provavelmente por inibir receptores de ocitocina no endométrio e diminuir a probabilidade da ocorrência de ciclos curtos (Zollers et al., 1993). 
Tabela 1 - Número de animais Nelore e mestiços (Nelore $\times$ Red Angus), proporção e porcentagem (entre parênteses) de vacas com corpo lúteo no início do tratamento, identificação do estro e prenhez em animais com 60 a 90 dias pós-parto submetidos a três tratamentos hormonais

Table 1 - Number of Nelore and crossbred (Nelore $\times$ Red Angus) animals, proportion and percentage (between brackets) of cows with corpus luteum (CL) at the beginning of treatment, estrus identification and pregnancy rate for animals from 60 and 90 days postpartum, by hormonal treatment

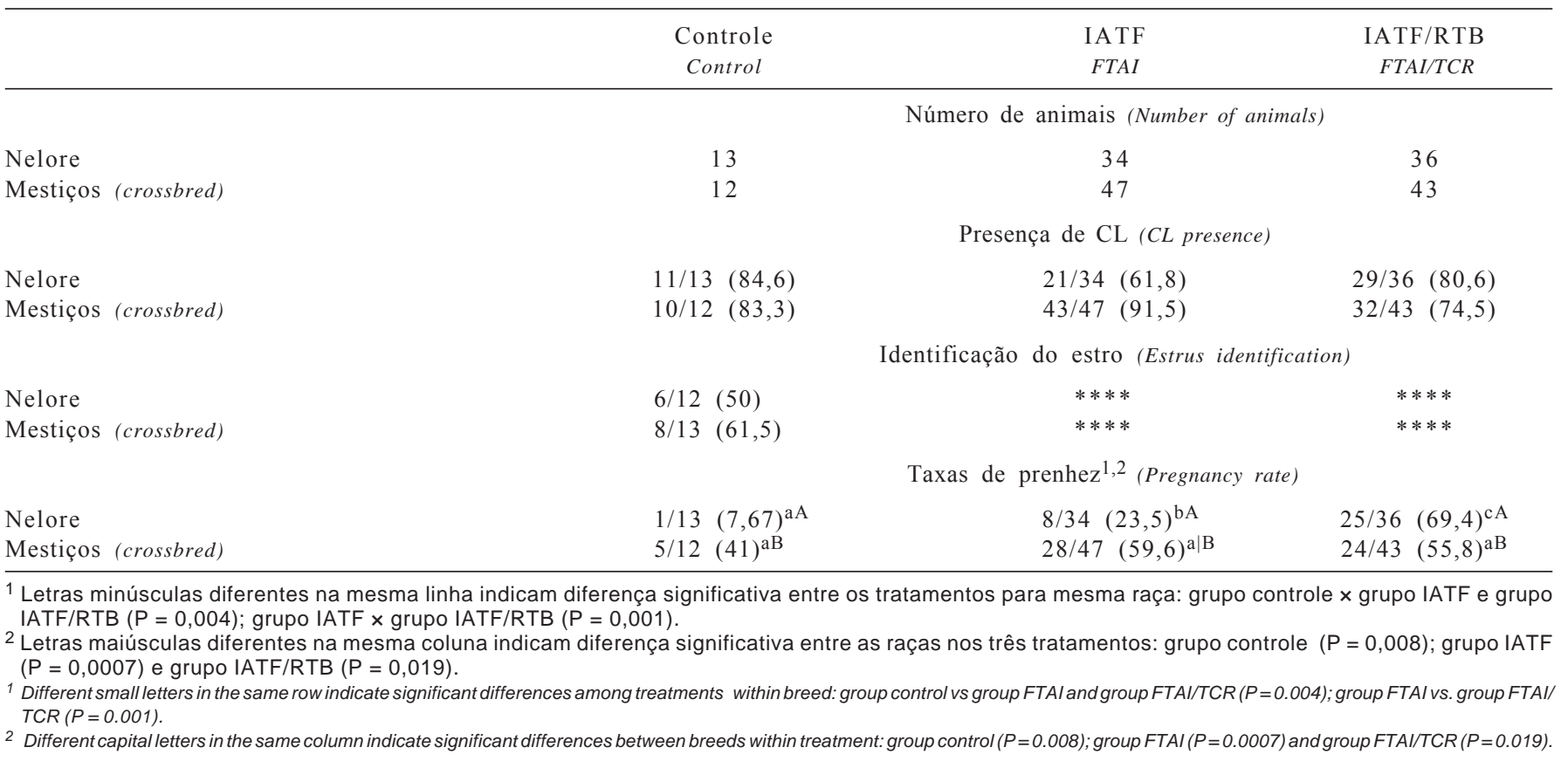

Tabela 2 - Taxa de prenhez (entre parênteses) de vacas Nelore aos 54 a 60 dias pós-parto submetidas a três tratamentos hormonais

Table 2 - Pregnancy rate (between brackets) of Nelore cows between 54 and 60 days postpartum, by hormonal treatment

\begin{tabular}{lccc}
\hline & IATF & IATF/RTB & IATF/eCG \\
& FTAI & FTAI/TCR & FTAI/eCG \\
\hline Prenhez $^{*}(\%)$ & $44 / 87(50,6)$ & $45 / 84(53,6)$ & $46 / 84(54,8)$ \\
Pregnancy (\%) & & & \\
\hline
\end{tabular}

* Não houve diferença significativa entre os três tratamentos $(P>0,05)$.

* Differences among treatments were not significant $(P>0.05)$.

Considerando que a RTB aumenta a pulsatilidade de LH em vacas acíclicas (Willians \& Griffith, 1995), a utilização de protocolos hormonais com progesterona ou progestágenos associados à RTB tem se mostrado eficiente em induzir o estro em vacas com 45 dias de parição, condição corporal 4 (escala de 1 a 9) e em anestro pósparto (Yelich et al., 1995; Firmino Neto et al., 1999). Meneghetii et al. (2001) verificaram que a remoção dos bezerros por 48 horas aumentou o tamanho do folículo dominante em vacas Nelore em anestro e que estas vacas apresentaram maior taxa de ovulação $(85,4 \%)$ à primeira aplicação de GnRH quando comparadas àquelas que não tiveram remoção de bezerros (taxa de ovulação de 51,0\%). Resultados similares foram descritos por Rivera et al. (1998), que observaram que a remoção temporária dos bezerros por 48 horas no momento da retirada do dispositivo intravaginal (D9) e a aplicação de GnRH 6 horas antes do retorno dos bezerros junto às suas mães resultou em taxa de ovulação de $86 \%$ nos animais tratados.

No experimento 1, a remoção temporária de bezerros (grupo IATF/RTB) promoveu aumento $(\mathrm{P}=0,001)$ na taxa de prenhez apenas nas vacas da raça Nelore, o que não ocorreu com as mestiças (Nelore $\times$ Red Angus). De forma similar, foi relatado que o efeito da RTB pode variar conforme a raça (Sanz et al., 2003) ou a idade das vacas (Geary et al., 2001; Santos \& Santos, 2003).

Uma possível explicação para o melhor desempenho da raça Nelore no tratamento IATF-RTB em relação às mestiças poderia ser a freqüência de mamadas. Segundo DAS et al. (2000), bezerros de vacas zebuínas apresentam maior freqüência e duração do ato de mamada, o que pode ser relacionado à baixa produção de leite de suas mães. Conseqüentemente, haveria maior inibição na liberação pulsátil de LH, retardando o crescimento folicular e diminuindo a taxa de ovulação. Entretanto, no experimento 2 a taxa de prenhez das vacas Nelore não melhorou quando os animais foram tratados com o protocolo IATF/RTB. Ressalta-se que as taxas de prenhez das vacas do grupo IATF já se encontravam em níveis satisfatórios (50,6\%), o que dificultou qualquer incremento adicional. Além disso, segundo Pires et al. (2004), as respostas reprodutivas 
obtidas com a RTB podem variar de acordo com a idade da vaca e do bezerro, com o nível nutricional ou até com o genótipo do rebanho, ou seja, dentro de uma mesma raça, algumas linhagens responderiam melhor à RTB que outras. Além disso, a linhagem das vacas Nelore utilizadas no experimento 1 (variedade pele rosa) difere da linhagem utilizada no experimento 2 .

A administração de eCG em vacas lactantes acíclicas tratadas com progesterona ou progestágenos, tem sido recomendada pela ação do FSH/LH no desenvolvimento dos folículos de vacas em anestro (Kastelic et al., 1999; Yavas e Walton, 2000b). Baruselli et al. (2003) encontraram maior $(55,1 \% \times 38.9 \%, \mathrm{P}<0,05)$ taxa de prenhez com uso de 400 UI de eCG em vacas Nelore lactantes e acíclicas tratadas com CIDR-B.Este efeito também foi observado por Cavalieri \& Fitzpatrick (1995) em novilhas Bos taurus indicus acíclicas (taxa de prenhez de 50\%) com adição de 400 UI de eCG quando retirados os implantes de norgestomet. Entretanto, neste trabalho, a associação de eCG ao protocolo IATF não aumentou a taxa de prenhez, possivelmente porque, além de os animais do grupo controle (IATF) apresentarem taxa de prenhez satisfatória $(50,6 \%)$, dificultando o aumento dessa taxa, a maioria das vacas Nelore do experimento 2 não se encontrava em anestro pós-parto e, portanto, não se beneficiou da aplicação desta gonadotrofina.

\section{Conclusões}

A retirada temporária do bezerro, associada a tratamentos hormonais com dispositivo intravaginal liberador de progesterona, aumenta a taxa de prenhez, enquanto a adição de eCG não melhora a taxa de prenhez de vacas Nelore lactantes ciclando e em boa condição corporal tratadas com dispositivos contendo progesterona.

\section{Literatura Citada}

ASSOCIAÇÃO BRASILEIRA DOS CRIADORES DE ZEBU ABCZ. [2003]. Disponível em: <htpp:\|www.abcz.org.br> Acesso em: $18 / 4 / 2006$.

ASSOCIAÇÃO OS CRIADORES DE NELORE DO BRASIL - ACNB. [2006]. htpp: \www.nelore.org.br Acesso em: 18/04/2006.

ANUALPEC. Anuário da pecuária brasileira. 10.ed. São paulo: Editora FNP, 2003. 400p.

BARROS, C.M.; FERNANDES, P. Inseminação artificial com tempo fixo em ebuínos. In: CONGRESSO BRASILEIRO DAS RAÇAS ZEBUÍNAS, 3., Uberaba. Anais... Uberaba: 1998. p. $112-127$.

BARROS, C.M.; MOREIRA, M.B.P.; FERNANDES, P. Manipulação farmacológica do ciclo estral para melhorar programas inseminação artificial ou de transferência de embriões. Arquivo Faculdade Veterinária, Sup1. 26, p.179-89, 1998.

BARUSELLI, P.S.; MARQUES, M.O.; NASSER, L.F et al. Effect of eCG on pregnancy rates of lactating zebu beef cows treated with CIDR-B devices for timed artificial insemination. Theriogenology, v.59, p.214 (abstract), 2003.

BARUSELLI, P.S.; REIS, E.L.; MARQUES, M.O et al. The use of hormonal treatments to improve reproductive performance of anestrous beef cattle in tropical climates. Animal Reproduction Science, v.82-83, p.479-486, 2004.

BELLOWS, R.A.; SHORT, R.E. Reproductive losses in beef industry. In: FIELDS, J.M.; SAND, R.S. (Eds.) Factors afecting calf crop. Boca Raton: CRC Press, 1994. p.109-133.

BÓ, G.A.; BARUSELli, P.S.; MARTINEZ, M.F. Pattern and manipulation of follicular development in Bos Indicus cattle. Animal Reproduction Science, v.78, p.307-326, 2003.

CAVALIERI, J.; FITZPATRICK, L.A. Oestrus detection techniques and insemination strategies in Bos indicus heifers synchronized with norgestomet oestradiol. Australian Veterinary Journal, v.72, p.177-182, 1995

COLÉGIO BRASILEIRO DE REPRODUÇÃO ANIMAL - CBRA. Manual para exame andrológico e avaliação de sêmen animal. 2.ed. Belo Horizonte: 1998. p.11-17.

CUBAS, A.C.; MANCIO, A.B.; LESSKIU, C. et al. Efeito da amamentação controlada sobre a eficiência reprodutiva de vacas de corte no sul do Paraná. Revista Brasileira de Zootecnia, v.14, p.247-255, 1985

DAS, S.M.; REDBO, I.; WIKTORSSON, H. Effect of age of calf on suckling behaviour and other behavioural activites of zebu and crossbred calves during restricted suckling periods. Applied Animal Behaviour Science, v.67, p.47-57, 2000.

DUNLAP, S.E.; KISER, T.E.; COX, N.M. et al. Cortisol and luteinizing hormone after adrecocorticotropic hormone administration to postpartum beef cows. Journal of Animal Science, v.52, p.587-593, 1981

FIRMINO NETO, J.E.; OLIVEIRA, M.A.L.; LIMA, P.F et al. A associação entre progestágenos e desmame temporário nos pós parto de vacas Nelore com cria ao pé. Revista Brasileira de Reprodução Animal, v.23, n.3, p.340-341, 1999.

GEARY, T.W.; WHITTIER, J.C.; HALLFORD, D.M. et al. Calf removal improves conception rates to the Ovsynch and CO-Synch protocols. Journal of Animal Science, v.79, p.1-4, 2001.

LOWMAN, B.G.; SCOTT, N.A.; SOMERVALLE, S.H. Condition scoring of cattle. Edinburgh: The East of Escotland College of Agriculture, 1976, p.1-13 (Bulletin, 6).

KASTELIC, J.P.; OLSON, W.O.; MARTINEZ, M.A. et al. Syncronization of estrus in beef cattle with norgestomet and estradiol valerate. Canadian Veterinary Journal, v.40, p.173-8, 1999.

MACMILLAN, K.L. Reproductive management. Champaing: Large Dairy Herd Management, 1992. p.88.

MENEGHETTI, M.; VILELA, E.R.; VASCONCELOS, J.L.M. et al. Efeito da remoção dos bezerros nos folículos dominante e na taxa de ovulação ao primeiro $\mathrm{GnRH}$ em protocolos de sincronização em vacas Nelore em anestro. Revista Brasileira de Reprodução Animal, v.25, n.3, p.286-288, 2001.

MIES FILHO, A. Reprodução dos animais e inseminação artificial. 4.ed. Porto Alegre Sulina, 1977, 652p.

NETT, T.M.; CERMAK, D.; BRADEN, T. et al. Pituitary receptors for GnRH and estradiol, and pituitary content of gonadotropins in beef cows. I Changes during the estrous cycle. Domestic Animal Endocrinology, v.4, p.123-132, 1987.

PIRES, V.A.; ARAUJO, C.R.; MENDES, Q.C. Fatores que interferem na eficiência reprodutiva de bovinos de corte. In: SIMPÓSIO PECUÁRIA INTENSIVA NOS TRÓPICOS. Anais... Piracicaba: Fundação de Estudos Agrários Luiz de Queiroz, 2004. p.355-398.

RIVERA, G.M.; GONI, C.G.; CHAVES, M.A. et al. Ovarian follicular wave synchronization and induction of ovulation in post-partum beef cows. Theriogenology, v.49, p.1365-1376, 1998.

SANTIAGO, A.A. O Zebu na Índia, no Brasil e no mundo. Campinas: Instituto Campineiro de Ensino Agrícola, 1985, 706p.

SANTOS, R. Nelore: a vitória brasileira. Uberaba: Agropecuária Tropical, 1995. 391p. 
SANTOS, F.A.P.; SANTOS, J.E.P. Efeitos da nutrição na reprodução de bovinos de corte em pastagens. In: REIS, R.A.; BERNARDES, T.F.; SIQUEIRA, G.R. et al. (Eds.) Volumosos na produção de ruminantes. Valor alimentício de forragens. 1.ed. Jaboticabal: Funep, 2003. p.123-164.

SANZ, A.; CASASÚS, I.; VILLALBA, D. et al. Effects of suckling frequency and breed on productive performance, follicular dynamics and postpartum interval in beef cows. Animal Reproduction Science, v.79, p.57-69, 2003.

STATISTICAL ANALYSIS SYSTEM - SAS. User's guide. Cary: 1999. (CD-ROM).

SAVIO, J.D.; THATCHER, W.W.; MORRIS, G.R. et al. Effects of induction of low plasma progesterone concentrations with a progesterone-releasing intravaginal device on follicular turnover and fertility in cattle. Journal Reproduction Fertility, v.98, p.77-84, 1993 .

SHIVELY, T.E.; WILliAMS, G.L. Patterns of tonic luteinizing hormone release and ovulation frequency in suckled anestrous beef cows following varying intervals of temporary weaning. Domestic Animal Endocrinology, v.6, p.379-387, 1989.

ZIMMER, A.H.; EUCLIDES FILHO, K. As pastagens e a pecuária de corte brasileira. In: SIMPÓSIO INTERNACIONAL SOBRE PRODUÇÃO ANIMAL EM PASTEJO, 1997, Viçosa, MG. Anais... Viçosa, MG: Universidade Federal de Viçosa, 1997. p.349-379.

ZOLLERS JR., W.G.; GARVERICK, H.A.; YOUNGQUIST, R.S. et al. In vitro secretion of prostaglandins from endometrium of postpartum beef cows expected to have short or normal luteal phases. Biology Reproduction, v.44, p.522-526, 1991.

ZOLLERS JR., W.G.; GARVERICK, H.A.; SMITH, M.F. et al. Concentrations of progesterone and oxytocin receptors in endometrium of postpartum cows expected o have a short or normal oestrus cycle. Journal Reproduction Fertility, v.97, p.329-337, 1993.
WALTERS, D.L.; SMITH, M.F.; HARMS, P.G. et al. Effect of steroids and $48 \mathrm{hr}$ calf removal on serum luteinizing hormone concentrations in anestrous beef cows. Theriogenology, v.18, p.349-356, 1982 .

WILLIANS, G.L.; GRIFFITH, M.K. Sensory and behavioural control of gonadotrophin secretion during suckling-mediated anovulation in cows. Journal Reproduction Fertility, v.49, p.463-475, 1995 (suppl.).

WILTBANK, J.N. Research needs in beef cattle reproduction. Journal of Animal Science, v.31, n.4, p.755-762, 1970.

WILTBANK, M.C.; GÜMEN, A.; SARTORI, R. Physiological classification of anovulatory conditions in cattle. Theriogenology, v.57, p.21-52, 2002.

YAVAS, Y.; WALTON, J.S. Induction of ovulation in postpartum suckled beff cows. A review. Theriogenology, v.54, n.1, p.123, 2000a.

YAVAS, Y.; WALTON, J.S. Postpartum acyclicity in suckled beff cows. A review. Theriogenology, v.54, n.1, p.24-55, $2000 \mathrm{~b}$.

YELICH, J.V.; HOLLAND, M.D.; SCHUTZ, D.N. et al. Synchronization of estrus in suckled postpartum beef cows with melengestrol acetate, 48 hour calf removal and PGF $2 \alpha$. Theriogenology, v.43, p.401-410, 1995. 